\title{
Elecciones presidenciales Perú 2011: ANÁLISIS COMPARATIVO DE LA ASOCIACIÓN ENTRE LOS RESULTADOS DE LA PRIMERA Y SEGUNDA VUELTA ELECTORAL A NIVEL MESA DE SUFRAGIO
}

\author{
Daniela Zacharías ${ }^{1}$ \\ David Sulmont ${ }^{2}$ \\ Gilda Garibotti ${ }^{3}$
}

Resumen: En la primera vuelta de las elecciones presidenciales peruanas de 2011, dos de cada cinco votos fueron para un candidato que no alcanzó a pasar a la segunda vuelta o ballotage. El objetivo de este trabajo es investigar cómo el electorado reorientó sus preferencias en la segunda vuelta entre los dos candidatos disponibles. Los datos utilizados son los resultados electorales a nivel de mesa de sufragio, la menor unidad de agregación posible. Para analizar la reorientación de votos se utilizaron modelos de regresión lineal múltiple tomando como variables dependientes la diferencia de los votos entre segunda y primera vuelta de los candidatos que pasaron al ballotage en cada mesa de sufragio. Se presentan análisis a nivel nacional, regional y por quintil de Î́ndice de Desarrollo Humano.

Palabras clave: trasvase de votos, modelo de regresión lineal múltiple, ballotage, elecciones.

\footnotetext{
1 Mg. en Estadística, consultora independiente, especializada en diseño muestral y observación electoral, Argentina. danielazach@gmail.com

2 Mg. en Sociología, director del Instituto de Opinión Pública y Profesor Principal del Dpto. de Ciencias Sociales, Pontificia Universidad Católica del Perú, Perú. sulmont@pucp.pe

3 Dra. en Estadística, Profesora Dpto. de Estadística, Universidad Nacional del Comahue, Argentina. gilda.garibotti@gmail.com
} 
Abstract: During the first round of the 2011 Peruvian presidential elections two out of five ballots went to candidates that would not take part in the second round (ballotage). The aim of this work is to identify how the citizens reoriented their electoral preferences between the two remaining candidates in the second round. The input data are the individual polling station election results i.e., the smallest available data units. Ballot reorientation was analyzed in terms of multiple linear regression models taking as dependent variables the vote difference between the second and first rounds for the candidates participating in the ballotage and each polling station. The results are analyzed at national and regional levels and by Human Development Index quintile.

Key words: Ballot reorientation, multiple linear regression models, ballotage, elections

\section{Introducción}

En abril de 2011 se llevó a cabo en Perú un proceso electoral en el cual ninguno de los candidatos presidenciales obtuvo el $50 \%$ más uno de los votos válidos. Por este motivo, tal como lo estipula la ley electoral peruana, se convocó a una segunda vuelta en junio del mismo año entre los dos candidatos con mayor votación: Ollanta Humala $(31,7 \%)$ y Keiko Fujimori (23,6\%).

Para muchos electores la decisión de voto en segunda vuelta no fue fácil, ya que el candidato de su preferencia había quedado fuera de carrera. Casi el $40 \%$ de los votos emitidos en la primera vuelta fue para alguno de los candidatos que no pasaron a la segunda vuelta. Estos electores tendrían que elegir entonces entre Keiko Fujimori (KF) y Ollanta Humala $(\mathrm{OH})$ o, en su defecto, tomar la opción de votar en blanco o anular el voto. Parte de la opinión pública consideraba que ésta sería una "elección imposible" entre dos grandes males (Vergara, 2011).

En la etapa previa a la segunda vuelta, los analistas políticos opinaban sobre cuál sería el comportamiento 
del electorado en este ballotage. Por ejemplo, el entonces director del Instituto de Opinión Pública de la Universidad Católica, Fernando Tuesta, señaló en una entrevista (Noriega, 2011) que no creía que los electores verían la elección de segunda vuelta como una disputa entre la izquierda, representada por Humala, y la derecha, que encarnaba Fujimori, ya que "en Perú, para la mayoría de votantes las elecciones no tienen que ver con derecha o izquierda. Las adhesiones son más personales que políticas o ideológicas". En esta misma entrevista, Carlos Monge, investigador del Centro de Estudios y Promoción del Desarrollo-DESCO, pronosticaba la siguiente trayectoria en el comportamiento electoral entre la primera y segunda vuelta:

Los sectores empresariales y de la clase media alta y alta votarían por Fujimori, igual que gran parte del electorado de $\mathrm{PPK}^{4}$ y el voto popular, de los sectores pobres, a favor de Castañeda, ${ }^{5}$ ya que es sociológicamente igual al de KF: es un voto clientelista, populista. El voto popular y de la clase media que fue a Toledo, ${ }^{6}$ y que tiene un nivel de preocupación por los derechos humanos y la democracia, podría ir a Humala si éste les da la señal de que el suyo sería un gobierno cercano a Lula y no a un estatismo chavista.

En general, otras hipótesis sobre cómo se daría el "trasvase" del voto, es decir, sobre cómo votarían los electorados de Pedro Pablo Kuczynski (PPK), de Alejandro Toledo (AT) y de Luis Castañeda (LC) en la segunda vuelta electoral, presentaban similitudes con las opiniones expresadas por Monge. Así también lo insinuaban las encuestas de intención de voto que se realizaron en este período, como la que se presenta en la Tabla 1 .

Pedro Pablo Kucynski. Obtuvo el tercer puesto en las elecciones de abril (18,5\% de los votos válidos).

5 Luis Castañeda. Ex Alcalde de Lima. Obtuvo el 9,8\% de los votos válidos (quinto lugar).

$6 \quad$ Alejandro Toledo. Ex Presidente. Obtuvo el cuarto lugar en las elecciones de abril, con un 15,6\% de los votos válidos. 
Tabla 1: Encuesta de intención de voto segunda vuelta electoral

\begin{tabular}{|c|c|c|c|c|c|c|}
\hline $\begin{array}{c}\text { Candidato } \\
\text { por el que } \\
\text { votó en } \\
\text { primera } \\
\text { vuelta }\end{array}$ & Humala & $\begin{array}{c}\mathrm{K} \\
\text { Fujimori }\end{array}$ & $\begin{array}{c}\text { Blanco/ } \\
\text { Viciado }\end{array}$ & $\begin{array}{c}\text { No sabe } \\
/ \text { No } \\
\text { respon- } \\
\text { de }\end{array}$ & Total & $\begin{array}{c}\text { Base de } \\
\text { entrevistas } \\
\text { ponderada }\end{array}$ \\
\hline Humala & $94 \%$ & $2 \%$ & $1 \%$ & $2 \%$ & $100 \%$ & 461 \\
\hline Fujimori & $3 \%$ & $94 \%$ & $1 \%$ & $3 \%$ & $100 \%$ & 364 \\
\hline Kuczynski & $18 \%$ & $50 \%$ & $20 \%$ & $12 \%$ & $100 \%$ & 290 \\
\hline Toledo & $36 \%$ & $28 \%$ & $21 \%$ & $16 \%$ & $100 \%$ & 165 \\
\hline Castañeda & $29 \%$ & $41 \%$ & $19 \%$ & $11 \%$ & $100 \%$ & 141 \\
\hline Otro & $63 \%$ & $37 \%$ & $0 \%$ & $0 \%$ & $100 \%$ & 3 \\
\hline $\begin{array}{c}\text { Blanco o } \\
\text { viciado }\end{array}$ & $11 \%$ & $6 \%$ & $55 \%$ & $28 \%$ & $100 \%$ & 48 \\
\hline No precisa & $24 \%$ & $39 \%$ & $13 \%$ & $25 \%$ & $100 \%$ & 17 \\
\hline
\end{tabular}

Fuente: Instituto de Opinión Pública - IOP.

El presente artículo tiene como objetivo evaluar, a la luz de los resultados oficiales de ambas elecciones, el grado en que la votación en la primera vuelta por PPK, AT y LC se asoció al voto por OH o por KF en la segunda vuelta electoral. También se consideró la influencia del voto no válido en la primera vuelta, ya que representa un porcentaje significativo mayor incluso al del candidato LC: $12,2 \%$ y $8,6 \%$ respectivamente.

Las hipótesis planteadas por distintos analistas políticos en relación con cómo se daría el trasvase de votos se basan en variables socioeconómicas y en la inclinación política de los votantes. Por ello, nos propusimos realizar un análisis cuantitativo para estudiar la validez de estas hipótesis, planteando un modelo estadístico que describa la manera en que se dio el trasvase, investigando la existencia de características diferenciales a nivel de región en el comportamiento electoral y la influencia en ese comportamiento de las variables sociodemográficas. 


\section{Data y metodología}

Los datos utilizados en este estudio son los resultados electorales, a nivel de mesa de sufragio, de las elecciones presidenciales de 2011 en primera y en segunda vuelta. En la elección hubo un total de 107.499 mesas de sufragio. En nuestro análisis no se incluyen las mesas de sufragio instaladas en el extranjero (3833 mesas), las mesas de sufragio anuladas en alguna de las dos elecciones (1223 mesas) ni aquéllas en las que el número de empadronados en la primera vuelta no coincide con el del ballotage (72 mesas). Como resultado de estas consideraciones, el universo de estudio comprendió 102.321 mesas de sufragio.

Se estudió la asociación entre la distribución de los votos en la primera y la segunda vueltas por medio de modelos de regresión lineal múltiple.

Variables dependientes consideradas:

- Diferencia de porcentajes de votos a favor de $\mathrm{OH}$ entre la segunda y la primera vuelta

- Diferencia de porcentajes de votos a favor de KF entre la segunda y la primera vuelta

Las variables independientes fueron los porcentajes de votos obtenidos en la primera vuelta por PPK, AT, LC y la diferencia de porcentajes de votos no válidos (blancos y nulos) entre ambas vueltas electorales.

Porcentajes, todos ellos, tomados sobre la base del total de votos emitidos en las respectivas vueltas electorales.

Los modelos considerados se expresan de la siguiente manera:

$$
\begin{aligned}
& \Delta H_{i}=\beta_{H, P P K} P P K_{i}+\beta_{H, A T} A T_{i}+\beta_{H, L C} L C_{i}+\beta_{H, N V} \Delta N V_{i}+\varepsilon_{H, i} \\
& \Delta F_{i}=\beta_{F, P P K} P P K_{i}+\beta_{F, A T} A T_{i}+\beta_{F, L C} L C_{i}+\beta_{F, N V} \Delta N V_{i}+\varepsilon_{F, i}
\end{aligned}
$$


Donde:

$\boldsymbol{i}=$ mesa de sufragio

$\mathrm{DH} \boldsymbol{i}=$ Diferencia (en \% de votos emitidos) de la votación por OH entre la segunda vuelta y la primera vuelta

$\mathrm{DF} \boldsymbol{i}=$ Diferencia (en \% de votos emitidos) de la votación por KF entre la segunda vuelta y la primera vuelta

$\boldsymbol{P P K i}=\%$ de votos emitidos obtenidos por PPK en la primera vuelta

$\boldsymbol{A T i}=\%$ de votos emitidos obtenidos por AT en la primera vuelta

$\boldsymbol{L C} \boldsymbol{i}=\%$ de votos emitidos obtenidos por LC en la primera vuelta

DNVi = Diferencia (en \% de votos emitidos) de votos no válidos (blancos y nulos) entre la segunda vuelta y la primera vuelta

$\varepsilon_{H, i}$ y $\varepsilon_{F, i}$ corresponden al término de error aleatorio para cada uno de los modelos

$\mathrm{b} \boldsymbol{H}, \boldsymbol{P P K}, \mathrm{bH}, \boldsymbol{A T}, \mathrm{bH}, \boldsymbol{L C}, \mathrm{bH}, \boldsymbol{N V}$, son los coeficientes lineales para las variables independientes PPK, AT, LC y NVi en la variación de \% de votos de $\mathrm{OH}$.

$\mathrm{b} \boldsymbol{F}, \boldsymbol{P P K}, \mathrm{b} \boldsymbol{F}, \boldsymbol{A T}, \mathrm{b} \boldsymbol{F}, \boldsymbol{L C}, \mathrm{b} \boldsymbol{F}, \boldsymbol{N} \boldsymbol{V}$, son los coeficientes lineales para las variables independientes PPK, AT, LC y NVi en la variación de \% de votos de KF.

De aquí en adelante, con el solo fin de hacer más fácil la lectura de este documento y a menos que se especifique lo contrario, nos referiremos al "porcentaje de votos emitidos" como el "porcentaje de votos".

Estos modelos se estimaron de manera global para las 102.321 mesas de sufragio consideradas así como por regiones geográficas y quintiles de Índice de Desarrollo Humano (PNUD, 2013).

Las regiones geográficas consideradas son: Lima (la capital del país), Costa Norte, Costa Centro, Costa Sur, Sierra Norte, Sierra Centro, Sierra Sur y Selva. Existen diferencias regionales en cuanto a variables socioeconómicas y demográficas que 
están claramente correlacionadas con esta diferenciación en el ámbito electoral. Las regiones de la sierra y de la selva tienen niveles de desarrollo socioeconómico menores que las de la costa; asimismo, estos niveles de desarrollo son mayores en las regiones del norte que en las del sur.

En el análisis de estos modelos de "trasvase" de votos entre primera y segunda vuelta resulta también de interés tomar en cuenta variables que reflejen diferencias socioeconómicas. Para representar esta dimensión hemos escogido el Índice de Desarrollo Humano (IDH) elaborado por el Programa de las Naciones Unidas para el Desarrollo (PNUD, 2013) por su significativa correlación con otras variables socioeconómicas (como por ejemplo el porcentaje de PEA asalariada y el de autoidentificación indígena) (Sulmont, 2009) y con el voto hacia determinados candidatos (El Comercio, 2011). El IDH resume una serie de indicadores vinculados con la formación de capital humano (en educación, condiciones de salud y recursos económicos) que hace posible comparar niveles de desarrollo social entre países y entre regiones o entre localidades de un mismo país.

A cada mesa de sufragio se le asignó el IDH del distrito al que pertenecía y fueron posteriormente agrupadas de acuerdo con los quintiles del IDH.

Todos los análisis estadísticos se realizaron utilizando el paquete estadístico $R$ versión 3.0.2. ( $R, 2013)$.

\section{Resultados}

\section{Resultados generales}

La Tabla 2 muestra los resultados de la primera vuelta de la elección para presidente de 2011 en Perú. Para cada candidato se presenta la cantidad de votos obtenidos y los porcentajes sobre votos válidos y emitidos. En la Tabla 3 se muestran los resultados de la segunda vuelta electoral. 
Tabla 2: Resultados elecciones presidenciales Perú 2011

\begin{tabular}{|c|c|c|c|c|}
\hline $\begin{array}{l}\text { Organización } \\
\text { Política }\end{array}$ & $\begin{array}{l}\text { Candidato } \\
\text { presidencial }\end{array}$ & Votos & $\begin{array}{c}\% \\
\text { sobre } \\
\text { votos } \\
\text { válidos }\end{array}$ & $\begin{array}{c}\% \text { sobre } \\
\text { votos } \\
\text { emitidos }\end{array}$ \\
\hline Gana Perú & Ollanta Humala & 4.643 .064 & $31,7 \%$ & $27,8 \%$ \\
\hline Fuerza 2011 & Keiko Fujimori & 3.449 .595 & $23,6 \%$ & $20,7 \%$ \\
\hline $\begin{array}{c}\text { Alianza Por El Gran } \\
\text { Cambio }\end{array}$ & $\begin{array}{l}\text { Pedro Pablo } \\
\text { Kuczynski }\end{array}$ & 2.711 .450 & $18,5 \%$ & $16,2 \%$ \\
\hline Perú Posible & Alejandro Toledo & 2.289 .561 & $15,6 \%$ & $13,7 \%$ \\
\hline $\begin{array}{c}\text { Alianza Solidaridad } \\
\text { Nacional }\end{array}$ & Luis Castañeda & 1.440 .143 & $9,8 \%$ & $8,6 \%$ \\
\hline $\begin{array}{c}\text { Partido Fonavistas } \\
\text { Del Peru }\end{array}$ & $\begin{array}{l}\text { José Antonio } \\
\text { Ñique }\end{array}$ & 37.011 & $0,3 \%$ & $0,2 \%$ \\
\hline Despertar Nacional & Ricardo Noriega & 21.574 & $0,1 \%$ & $0,1 \%$ \\
\hline Adelante & Rafael Belaunde & 17.301 & $0,1 \%$ & $0,1 \%$ \\
\hline Fuerza Nacional & Juliana Reymer & 16.831 & $0,1 \%$ & $0,1 \%$ \\
\hline $\begin{array}{c}\text { Justicia Tecnología Y } \\
\text { Ecología }\end{array}$ & $\begin{array}{c}\text { Humberto } \\
\text { Pinazo }\end{array}$ & 11.275 & $0,1 \%$ & $0,1 \%$ \\
\hline $\begin{array}{l}\text { P.descentralista } \\
\text { Fuerza Social }\end{array}$ & Renunció & 9.358 & $0,1 \%$ & $0,1 \%$ \\
\hline $\begin{array}{l}\text { Total De Votos } \\
\text { Válidos }\end{array}$ & & 14.647 .163 & $100,0 \%$ & $87,7 \%$ \\
\hline Votos Blancos & & 1.477 .696 & & $8,8 \%$ \\
\hline Votos Nulos & & 574.875 & & $3,4 \%$ \\
\hline $\begin{array}{l}\text { Total De Votos } \\
\text { Emitidos }\end{array}$ & & 16.699 .734 & & $100,0 \%$ \\
\hline $\begin{array}{c}\text { Total De Electores } \\
\text { Hábiles }\end{array}$ & & $\mid 19.949 .915$ & & \\
\hline
\end{tabular}

Fuente: ONPE, 2011 a. 
Tabla 3: Resultados elecciones presidenciales Perú 2011 - Segunda vuelta

\begin{tabular}{|c|c|c|c|c|}
\hline $\begin{array}{c}\text { Organización } \\
\text { Política }\end{array}$ & $\begin{array}{c}\text { Candidato } \\
\text { presidencial }\end{array}$ & Votos & $\begin{array}{c}\% \\
\text { sobre } \\
\text { votos } \\
\text { válidos }\end{array}$ & $\begin{array}{c}\% \text { sobre } \\
\text { votos } \\
\text { emitidos }\end{array}$ \\
\hline Gana Perú & Ollanta Humala & 7.937 .704 & $51,5 \%$ & $48,2 \%$ \\
\hline Fuerza 2011 & Keiko Fujimori & 7.490 .647 & $48,5 \%$ & $45,5 \%$ \\
\hline $\begin{array}{c}\text { Total de votos } \\
\text { válidos }\end{array}$ & & 15.428 .351 & $100,0 \%$ & $93,7 \%$ \\
\hline Votos blancos & & 116.335 & & $0,7 \%$ \\
\hline Votos nulos & & 921.711 & & $5,6 \%$ \\
\hline $\begin{array}{c}\text { Total de votos } \\
\text { emitidos }\end{array}$ & & 16.466 .397 & & $100,0 \%$ \\
\hline & & 19.949 .915 & & \\
\hline $\begin{array}{c}\text { Total de electores } \\
\text { hábiles }\end{array}$ & & & & \\
\hline
\end{tabular}

Fuente: ONPE, 2011 b.

Entre la primera y la segunda vuelta electoral, el porcentaje de votos para $\mathrm{OH}$ aumentó de $27,8 \%$ a 48,2\% mientras que el de KF creció de 20,7\% a 45,5\%. Cabe observar que el incremento porcentual de votos a favor de KF superó al recibido por $\mathrm{OH}$. Sin embargo, este crecimiento superior no alcanzó para darle el triunfo a dicha candidata. En la Figura 1 se grafica la distribución de frecuencias de estos incrementos para ambos candidatos. 
Figura 1: Distribución de la diferencia de porcentajes de votos entre vueltas, por mesa de sufragio

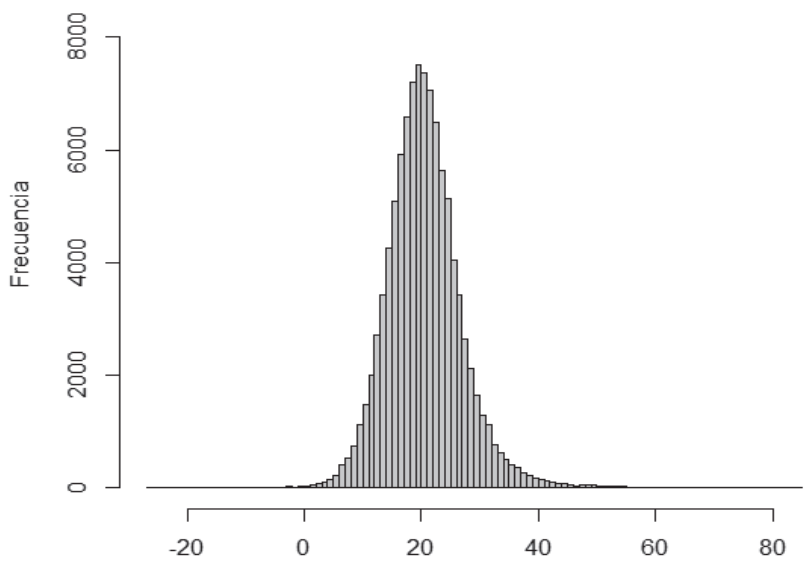

Diferencia en porcentajes de votos entre vueltas - HUMALA

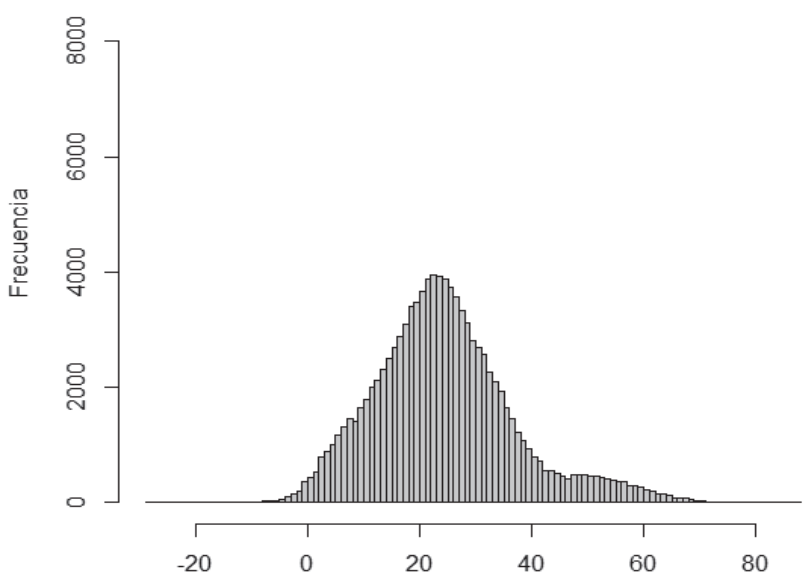

Diferencia en porcentajes de votos entre vueltas - FUJIMORI

Ajustando los modelos representados por las ecuaciones (1) y (2) mediante el método de regresión lineal múltiple aplicado 
a los datos individuales por mesa, se obtiene los coeficientes que se presentan en la Tabla 4.

Tabla 4. Estadísticos modelos de regresión

\begin{tabular}{|c|c|c|}
\cline { 2 - 3 } \multicolumn{1}{c|}{} & \multicolumn{2}{c|}{ Variable dependiente } \\
\hline Coeficientes* $^{*}$ & $\Delta \boldsymbol{H}$ & $\Delta \boldsymbol{F}$ \\
\hline bPPK & 0,20 & 0,80 \\
\hline $\mathrm{b} A T$ & 0,63 & 0,38 \\
\hline $\mathrm{b} L C$ & 0,50 & 0,52 \\
\hline $\mathrm{b} N V$ & 0,65 & 0,38 \\
\hline$R 2$ ajustado & 0,9360 & 0,9614 \\
\hline
\end{tabular}

*Todos los coeficientes son significativos, $p<0,001$

De acuerdo con estos resultados, cada punto en el porcentaje de votos obtenidos por PPK en la primera vuelta se asocia con un crecimiento de 0.8 en la diferencia de porcentajes de votos recibidos por KF mientras que sólo de 0.2 en los de $\mathrm{OH}$. Por otro lado, los votos de LC influyen de manera similar en los dos candidatos, mientras que los de AT y los no válidos favorecen en mayor medida a $\mathrm{OH}$ que a $\mathrm{KF}$, dado que el aumento de un punto en el porcentaje de votos obtenidos por AT o en los votos no válidos produciría un aumento de 0.6 en la diferencia en el porcentaje de votos de $\mathrm{OH}$ y de 0.4 en el de KF. Estos resultados se presentan en forma gráfica en la Figura 2. 
Figura 2: Coeficientes de regresión de los modelos de regresión 1 y 2
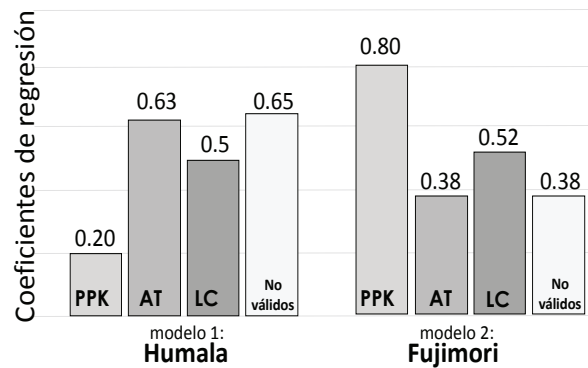

\section{Análisis por región}

La Tabla 5 presenta los promedios de los porcentajes de votos a nivel de mesa electoral para la primera y la segunda vuelta electoral por región.

Tabla 5. Promedio de porcentajes de votos en las mesas de sufragio de cada región

\begin{tabular}{|c|c|c|c|c|c|c|c|c|c|}
\hline \multirow{2}{*}{\multicolumn{2}{|c|}{ Candidato }} & \multirow{2}{*}{ Lima } & \multicolumn{3}{|c|}{ Costa } & \multicolumn{3}{|c|}{ Sierra } & \multirow{2}{*}{ Selva } \\
\hline & & & Norte & Centro & Sur & Norte & Centro & Sur & \\
\hline \multirow{6}{*}{$\begin{array}{c}1^{\mathrm{a}} \\
\text { vuelta }\end{array}$} & $\mathrm{OH}$ & 19,1 & 24,8 & 26,0 & 36,2 & 22,3 & 34,9 & 49,2 & 32,4 \\
\hline & $\mathrm{KF}$ & 20,0 & 25,2 & 30,5 & 19,8 & 24,5 & 18,7 & 12,5 & 24.4 \\
\hline & PPK & 26,1 & 11,5 & 13,7 & 15,9 & 5,1 & 11,2 & 13,0 & 6,4 \\
\hline & AT & 14,8 & 13,5 & 10,6 & 11,3 & 23,0 & 15,1 & 7,3 & 15,8 \\
\hline & LC & 12,2 & 13,1 & 8,6 & 8,5 & 3,2 & 4,6 & 4,0 & 3,4 \\
\hline & $\begin{array}{l}\text { No } \\
\text { válido }\end{array}$ & 7,2 & 11,3 & 10,1 & 7,8 & 21,1 & 14,7 & 13,1 & 17,0 \\
\hline \multirow{3}{*}{$\begin{array}{c}2^{\mathrm{a}} \\
\text { vuelta }\end{array}$} & $\mathrm{OH}$ & 39,8 & 44,7 & 43,2 & 55,0 & 46,3 & 57,4 & 68,8 & 53,4 \\
\hline & KF & 54,3 & 49,4 & 52,0 & 40,6 & 45,7 & 36,9 & 25,3 & 41,4 \\
\hline & $\begin{array}{c}\text { No } \\
\text { válido }\end{array}$ & 5,9 & 5,9 & 4,8 & 4,4 & 7,9 & 5,6 & 5,7 & 5,1 \\
\hline
\end{tabular}


Se observa que en la primera vuelta $\mathrm{OH}$ se impuso en las regiones Costa Sur, Sierra Centro y Sur, y Selva. Por su parte, KF lo hizo en Costa Norte y Centro, y Sierra Norte. Finalmente, PPK se impuso en Lima. En la segunda vuelta se mantiene esta estructura salvo en Lima, donde KF toma el primer lugar, y en la Sierra Norte, donde el primer lugar en el porcentaje de votos obtenidos pasa de $\mathrm{KF}$ a OH. Como se verá más adelante, $\mathrm{OH}$ captó la mayor parte de los votos de AT en esta última región, en la cual AT había obtenido su mejor resultado en la primera vuelta $(23 \%)$.

Para comprender el impacto que estas diferencias regionales puedan tener a nivel global es necesario tomar en cuenta el peso que éstas tienen en cuanto a la población electoral. En la Figura 3 se puede observar que más de la tercera parte de los votos emitidos corresponden a la región de Lima, mientras que la Costa abarca el 24\%, la Sierra el $30 \%$ y la Selva sólo el $10 \%$.

Figura 3: Distribución de los votos emitidos por región

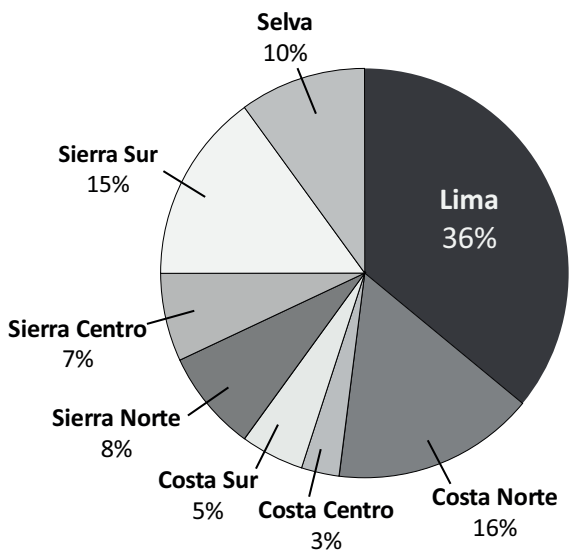


Analizando a nivel regional las diferencias entre los porcentajes de votos para OH y KF entre vueltas, podemos apreciar en la Tabla 6 que el crecimiento de KF fue más pronunciado en Lima y menos en la Costa. Por su parte, Humala obtuvo un crecimiento homogéneo en todas las regiones consideradas y superó a su contrincante en las zonas de Sierra y Selva. La ventaja que $\mathrm{OH}$ ya tenía en la Sierra y la Selva respecto de KF se amplía de manera significativa en la segunda vuelta con el trasvase de votos; eso, junto al hecho de que logra mejorar su posición en lugares estratégicos como la Costa Sur, le permite superar a su contrincante en el conteo final nacional de votos, a pesar de que KF obtuvo la mayoría de votos en las dos regiones demográficamente más importantes (y más desarrolladas en términos económicos) del país: Lima y la Costa Norte.

Tabla 6: Diferencia entre porcentajes de votos emitidos entre vueltas por región

\begin{tabular}{|c|c|c|c|c|c|c|c|c|}
\hline $\begin{array}{c}\text { Diferencia } \\
\text { entre } \\
\text { porcen- } \\
\text { tajes }\end{array}$ & Lima & \multicolumn{3}{|c|}{ Costa } & \multicolumn{3}{c|}{ Sierra } & \\
\cline { 3 - 9 } & Norte & Centro & Sur & Norte & Centro & Sur & Selva \\
\hline OH & 20,6 & 19,8 & 17,2 & 18,8 & 24,0 & 22,4 & 19,6 & 21,0 \\
\hline KF & 34,3 & 24,2 & 21,5 & 20,8 & 21,1 & 18,2 & 12,9 & 17,0 \\
\hline OH-KF & $-13,7$ & $-4,4$ & $-4,3$ & $-2,0$ & 2,9 & 4,2 & 6,7 & 4,0 \\
\hline
\end{tabular}

Los coeficientes de ajuste lineal del modelo 1 y el modelo 2 se grafican en las Figuras 4 y 5 respectivamente. De manera general se observa un importante trasvase de votos de AT hacia $\mathrm{OH}$, quien además recibió aproximadamente la mitad de la diferencia entre primera y segunda vuelta de los votos no válidos. En Lima, en la Selva y en mayor medida en la Sierra Sur, el trasvase de votos de LC favorece a $\mathrm{OH}$. Una situación peculiar se observa en la Sierra Sur, donde casi la totalidad de votos a favor de AT y 
LC, así como la diferencia de votos no válidos, favorecieron a $\mathrm{OH}$ en segunda vuelta. La votación por PPK muestra, para todas las regiones, un mayor impacto en el aumento de la diferencia de porcentaje de votos obtenidos en la segunda vuelta por KF, sobre en todo en Lima, donde KF obtuvo en forma mayoritaria los votos de PPK. La segunda fuente importante de votos adicionales para KF se ubica en quienes eligieron inicialmente a LC, en particular en las zonas de Costa, y Sierra Centro-Norte. Comparativamente, KF tuvo poca repercusión entre los votantes de AT y los de la región Sierra Sur, y es probable que esto le haya costado el triunfo en segunda vuelta.

Figura 4. Coeficientes de regresión para modelo 1, según región

Variable dependiente:

Diferencia en \% de votos obtenidos por HUMALA entre la segunda y la primera vuelta

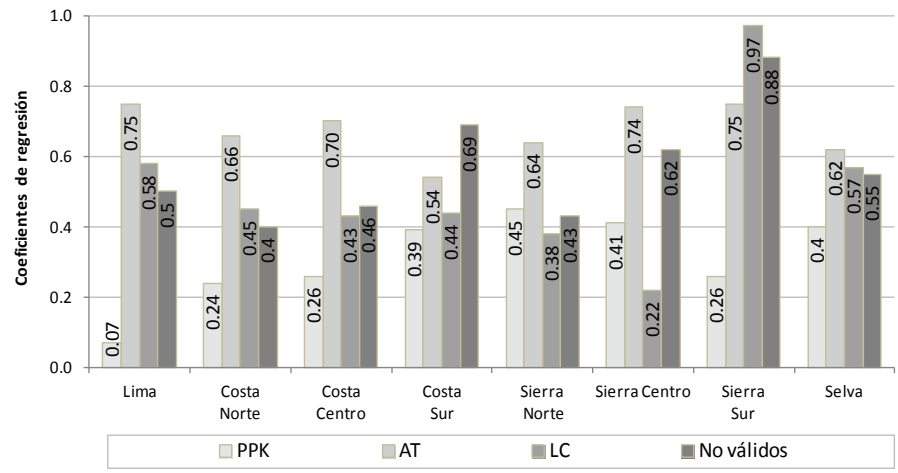

*Todos los coeficientes son significativos, $p<0.001$. R2 ajustado $>0.9$ 
Variable dependiente:

Diferencia en \% de votos obtenidos por FUJIMORI entre la segunda y la primera vuelta

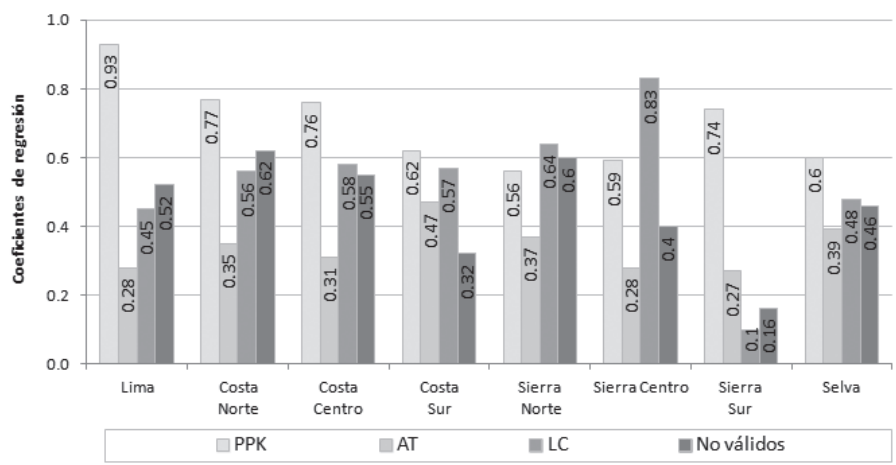

*Todos los coeficientes son significativos, $p<0.001$. R2 ajustado $>0.9$

\section{Análisis según Î́ndice de Desarrollo Humano (IDH)}

La Tabla 7 presenta los promedios de los porcentajes de votos a nivel de mesa electoral para la primera y la segunda vuelta electoral por quintil de IDH. 
Tabla 7. Promedio de porcentajes de votos en las mesas de sufragio en cada quintil de IDH

\begin{tabular}{|c|c|c|c|c|c|c|}
\hline \multicolumn{2}{|c|}{ Candidato } & \multicolumn{5}{|c|}{ IDH } \\
\cline { 3 - 7 } & $\begin{array}{c}\text { Quintil } \\
1\end{array}$ & $\begin{array}{c}\text { Quintil } \\
2\end{array}$ & $\begin{array}{c}\text { Quintil } \\
3\end{array}$ & $\begin{array}{c}\text { Quintil } \\
4\end{array}$ & $\begin{array}{c}\text { Quintil } \\
5\end{array}$ \\
\hline \multirow{4}{*}{ 1a vuelta } & $\mathrm{OH}$ & 37,0 & 31,8 & 31,2 & 25,5 & 15,7 \\
\cline { 2 - 7 } & $\mathrm{KF}$ & 20,9 & 26,0 & 19,8 & 21,7 & 15,5 \\
\cline { 2 - 7 } & $\mathrm{PPK}$ & 2,5 & 8,5 & 15,7 & 19,3 & 34,8 \\
\cline { 2 - 7 } & $\mathrm{AT}$ & 14,6 & 12,2 & 13,9 & 13,1 & 16,1 \\
\cline { 2 - 7 } & $\mathrm{LC}$ & 2,5 & 8,1 & 9,7 & 11,8 & 11,5 \\
\cline { 2 - 7 } & No válido & 21,8 & 12,8 & 9,0 & 7,9 & 6,0 \\
\hline \multirow{3}{*}{$2^{\text {a vuelta }}$} & $\mathrm{OH}$ & 58,1 & 50,8 & 52,4 & 46,3 & 36,4 \\
\cline { 2 - 7 } & $\mathrm{KF}$ & 35,1 & 44,0 & 42,2 & 48,2 & 56,9 \\
\cline { 2 - 7 } & No válido & 6,6 & 5,1 & 5,4 & 5,4 & 6,6 \\
\hline
\end{tabular}

Se observa la relación inversa en el voto por Humala según aumenta el IDH distrital. El porcentaje de votos por $\mathrm{OH}$ en el quintil inferior más que duplica el alcanzado en el quintil superior en la primera vuelta, y aún en la segunda es significativamente mayor. En la primera vuelta se observa que el porcentaje de votos por PPK se incrementa conforme aumenta el IDH distrital del elector. La distribución del voto favorable a KF muestra semejante comportamiento sólo en la segunda vuelta. A partir de esta segmentación se puede observar que en la primera vuelta disminuye la cantidad de votos no válidos conforme aumenta el IDH, mientras que en la segunda vuelta el porcentaje de votos no validos es homogéneo entre quintiles de IDH. Esto podría deberse a la mayor complejidad que presentó la cédula de votación en la primera vuelta, al comprender elecciones para Presidente, Congreso (con opción por el voto preferencial) y Parlamento Andino, en comparación con la segunda vuelta en la cual sólo figuraban dos candidatos. 
Analizando las variaciones de los porcentajes de votos para $\mathrm{OH}$ y KF entre vueltas (Tabla 8), se observa una tendencia al crecimiento del voto de KF en los sectores sociales altos y medio-alto, la cual, junto con el crecimiento de $\mathrm{OH}$ relativamente parejo a lo largo de todo el espectro social, resulta en un aumento pronunciado en la diferencia de porcentajes de votos ganados por cada candidato conforme aumenta el IDH a favor de KF, quien logra en el quintil superior duplicar el crecimiento que tuvo $\mathrm{OH}$.

Tabla 8: Diferencia entre porcentajes de votos emitidos entre vueltas por quintil de IDH

\begin{tabular}{|c|c|c|c|c|c|}
\hline \multirow{2}{*}{$\begin{array}{c}\text { Diferencia entre } \\
\text { porcentajes }\end{array}$} & \multicolumn{5}{|c|}{ IDH } \\
\cline { 2 - 6 } & $\begin{array}{c}\text { Quintil } \\
1\end{array}$ & $\begin{array}{c}\text { Quintil } \\
2\end{array}$ & $\begin{array}{c}\text { Quintil } \\
3\end{array}$ & $\begin{array}{c}\text { Quintil } \\
4\end{array}$ & $\begin{array}{c}\text { Quintil } \\
5\end{array}$ \\
\hline $\mathrm{OH}$ & 21,2 & 19,0 & 21,2 & 20,8 & 20,8 \\
\hline $\mathrm{KF}$ & 14,2 & 18,0 & 22,3 & 26,5 & 41,5 \\
\hline $\mathrm{OH}-\mathrm{KF}$ & 7,0 & 1,0 & $-1,1$ & $-5,7$ & $-20,7$ \\
\hline
\end{tabular}

En las Figuras 5 y 6 se grafican los coeficientes de ajuste lineal del modelo 1 y del modelo 2 respectivamente. Se puede observar, por un lado, que el comportamiento de quienes votaron a AT y de quienes habiendo emitido inicialmente votos no válidos optaron por un candidato en la segunda vuelta, es relativamente uniforme para todo el espectro social en relación con el apoyo a OH. Los votantes de PPK, por su parte, muestran comportamientos diferenciados habiendo optado por $\mathrm{OH}$ en relación inversa a su IDH. Mientras que una mayoría de votantes de primer quintil de IDH apoyó a $\mathrm{OH}$ en segunda vuelta, muy pocos de aquellos con altos ingresos (Quintil 5 de IDH) lo hicieron así. Tal como sucede con el análisis regional de Lima, la mayor parte de quienes votaron a PPK se encuentran en este último segmento, lo cual explica el bajo nivel de votación 
global de $\mathrm{OH}$ entre votantes de PPK. Finalmente, el peso de los electores de LC en el aumento del porcentaje de votos obtenidos por $\mathrm{OH}$ entre la segunda y la primera vuelta es, en cierta medida, inverso al del electorado de PPK.

En el caso del trasvase de votos hacia KF, se observa una mayor preferencia hacia esta candidata entre los votantes de PPK (con menor grado de aceptación en el Quintil 1). También se observa un trasvase preferencial desde los votantes de LC, aunque esto ocurre en menor medida en el Quintil 5. La votación de AT derivada a KF en segunda vuelta también se mantiene pareja (aunque en menor nivel que la obtenida por $\mathrm{OH}$ ) a lo largo del espectro del índice de desarrollo.

Figura 5. Coeficientes de regresión para modelo 1, según quintil de IDH

Variable dependiente:

Diferencia en \% de votos obtenidos por HUMALA entre la segunda y la primera vuelta

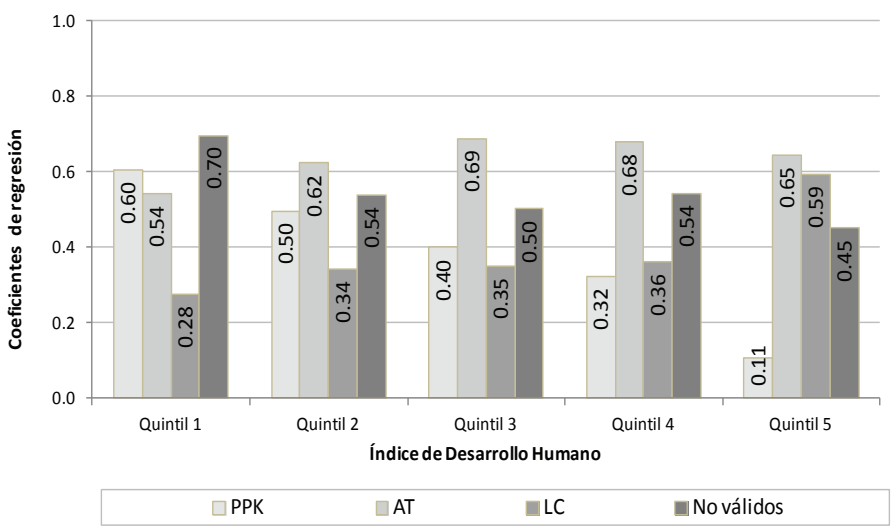

${ }^{*}$ Todos los coeficientes son significativos, $p<0.001$. R2 ajustado $>0.9$ 
Figura 6. Coeficientes de regresión para modelo 2 , según quintil de IDH

Variable dependiente:

Diferencia en \% de votos obtenidos por FUJIMORI entre la segunda y la primera vuelta

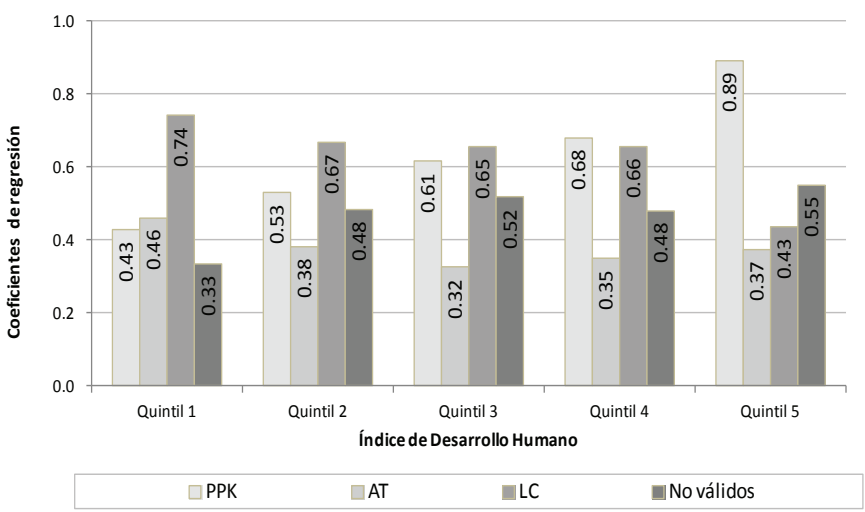

*Todos los coeficientes son significativos, $p<0.001$. R2 ajusta$d o=0.8$ en Quintil 1, en resto $>0.9$.

\section{Discusión}

El análisis de los modelos tiende a confirmar las hipótesis del trasvase de votos entre la primera y la segunda vuelta formuladas por los diversos analistas políticos peruanos. En las mesas de sufragio donde Alejandro Toledo obtuvo mejores resultados en la primera vuelta, los resultados de Ollanta Humala fueron netamente mucho más favorables que los de Keiko Fujimori en la segunda vuelta. Lo inverso ocurre con el voto por Kuczynski en primera vuelta. En aquellas mesas donde este candidato obtuvo mejores resultados, la tendencia general fue que Keiko Fujimori tuviera en la segunda vuelta mayor ganancia de votos que Ollanta Humala. En el caso de los votos por Castañeda, los modelos 
indican que se habrían trasvasado mayoritariamente a favor de Keiko Fujimori. Con respecto al voto blanco y nulo, se observa que en las mesas con mayor porcentaje de votos no válidos en la primera vuelta con respecto a la segunda, dicho diferencial de votación se dirigió preferentemente a Humala. Este patrón se reproduce si controlamos los modelos por región geográfica, aunque cambia la intensidad de los coeficientes. En las regiones que constituyen "bastiones electorales" de los respectivos candidatos (Lima para KF y la Sierra Sur para $\mathrm{OH}$ ), el trasvase de votos en el sentido indicado en los modelos se hace más intenso (en Lima, casi todos los votos obtenidos por PPK se habrían inclinado por KF y en la Sierra Sur los votos de LC favorecerían a OH).

Cuando se controla por Índice de Desarrollo Humano, se observan algunos cambios interesantes en el patrón general. En las mesas de sufragio de localidades que se ubican en el quintil superior del IDH, el efecto del voto por PPK en primera vuelta sobre el voto por KF en la segunda es muy fuerte, casi todos los votos de PPK en este tipo de lugares se orientaron hacia KF, lo que es consistente con lo afirmado en el párrafo anterior ya que casi todas las localidades de Lima se ubican en este quintil. Sin embargo, en los quintiles inferiores el voto por PPK se trasvasó más hacia $\mathrm{OH}$ que hacia KF, lo que indicaría que en este tipo de localidades (muchas de ellas ubicadas en la Sierra, y en la Sierra Sur en particular), habrían pesado más las consideraciones de identificación "social" con las candidaturas, que aquellas relacionadas con orientaciones ideológicas o programáticas.

En general los modelos estimados parecen reforzar las hipótesis de las teorías espaciales del comportamiento electoral, es decir que los electores evalúan y comparan sus preferencias políticas con la oferta electoral y votan por aquellas opciones que se acercan más a sus propias preferencias. 
Entre los analistas políticos peruanos, a pesar de las críticas y del escepticismo que existe sobre la utilidad de la dimensión izquierda-derecha como variable relevante para comprender el comportamiento electoral, habría un cierto consenso en la forma en que se posicionarían las candidaturas presentadas en primera vuelta en un eje izquierda-derecha: Humala estaría más inclinado hacia la izquierda; Toledo hacia el centro; Castañeda hacia el centro derecha y Kuczynski y Fujimori hacia la derecha en posiciones muy cercanas.

Los patrones de trasvase de votos identificados en estos modelos apuntarían a que los electores que inicialmente manifestaron su preferencia por candidatos que no lograron superar la barrera electoral para pasar a segunda vuelta, se inclinaron hacia el candidato que aparecía más próximo en el espectro político. Humala se habría beneficiado de un voto "centrista" expresado inicialmente a favor de Toledo y Castañeda, tendencia que fue reforzada por su propia estrategia de campaña destinada a "correrse al centro", tanto discursiva como programáticamente; en tal sentido, empleó una estrategia de campaña diametralmente opuesta a la utilizada en las elecciones de 2006.

Por su parte, la estrategia de campaña de segunda vuelta de Fujimori no logró el objetivo de posicionarse suficientemente hacia el centro para ganar esos votantes, y tan solo logró atraer a los votantes de la alternativa que se encontraba más próxima en la primera vuelta (la de PPK), lo cual no alcanzó para superar a Humala.

$\mathrm{Al}$ controlar los modelos por región e IDH, las tendencias generales no cambian sino que se ven reforzadas por las identificaciones sociales que existen entre ciertas localidades y las opciones políticas. La única excepción es el voto por PPK en las localidades de mayor pobreza, lo que indicaría que en este tipo de lugares el razonamiento 
"espacial" (más político) del votante choca o es interferido por otras consideraciones de mayor identificación social.

Si esta interpretación de los resultados fuera adecuada, pondría en cuestión las afirmaciones de que los votantes son personas poco sofisticadas y que no deciden su voto tomando en cuenta consideraciones de tipo político (ideológico o programático) y que privilegian más bien las identificaciones personales. Tal vez el elector peruano no tenga un nivel de sofisticación política muy elevado, y en la primera vuelta las identificaciones personales pueden tener un peso importante, pero cuando las opciones se restringen (como en una segunda vuelta) sí parece tener la suficiente capacidad para aplicar una racionalidad "espacial" (identificar las alternativas más cercanas a sus preferencias políticas) para tomar una decisión.

Esto nos lleva a reevaluar la utilidad de la dimensión izquierda-derecha en el análisis del comportamiento electoral. Más que posiciones fijas con contenidos precisos tanto en lo ideológico como en lo programático, la dimensión izquierdaderecha se convierte en un atajo cognitivo o en una herramienta de heurística política que le permite al elector clasificar o hacer un mapa simple de la oferta electoral en función de la posición que tienen los actores políticos respecto de algunos de los temas clave para las sociedades y que definen el contenido del debate electoral en un momento determinado.

\section{Agradecimientos}

Se agradece a Katiuska Valencia, Subgerente de Procesamiento del Registro Electoral (RENIEC) y a Piero Corvetto, Gerente del Registro Electoral (RENIEC) por habernos facilitado la base de datos; a Beniamín Zacharías por la adaptación de esa base para su análisis y a Alberto Baruj por la revisión final. 


\section{Bibliografía}

El Comercio (2011), “¿Cómo se moverán los votos en segunda vuelta?: la batalla será por Lima", Diario El Comercio, 17 de abril. Disponible en línea: http://elcomercio.pe/ politica/gobierno/como-se-moveran-votos-segundavuelta-batalla-lima-noticia-744155.

IOP (2011), Encuesta Nacional Urbana Rural. Intención de voto presidencial 2011 Segunda Vuelta, Pontificia Universidad Católica del Perú. Disponible en línea: http://es.scribd.com/doc/54915365/2011-Intencionde-voto-presidencial-segunda-vuelta-mayo-Nacional.

Noriega, C. (2011). "La derecha cavernaria apoyará a Keiko", Pagina 12, 12 de abril. Disponible en línea: http://www.pagina12.com.ar/diario/elmundo/4-166059-2011-04-12.html.

ONPE (2011a). Resultados Elecciones Generales y de Parlamento Andino 2011. ONPE (Oficina Nacional de Procesos Electorales). Disponible en línea: http:// www.web.onpe.gob.pe/modElecciones/elecciones/ elecciones2011/1ravuelta/.

ONPE (2011b), Resultados Eleccciones Generales 2011 Segunda Elección Presidencial. ONPE (Oficina Nacional de Procesos Electorales). Disponible en línea: http:// www.web.onpe.gob.pe/modElecciones/elecciones/ elecciones2011/2davuelta/.

PNUD (2013). Informe sobre Desarrollo Humano (IDH) Perú 2013. Cambio climático y territorio: Desafíos y respuestas para un futuro sostenible. Programa de las Naciones Unidas para el desarrollo. Disponible en línea: http://www.undp.org/content/peru/es/home/ library/poverty/Informesobredesarrollohumano2013/ IDHPeru2013/. 
R Development Core Team (2013). R: A Language and Environment for Statistical Computing. Viena: R Foundation for Statistical Computing, 2013.

Sulmont, D. (2009). "Líneas de frontera y comportamiento electoral en el Perú. Diferencias sociales y tendencias del voto en las elecciones presidenciales peruanas: 1980-2006". En: Plaza, O. Cambios sociales en el Perú 1968-2008. Lima: PUCP. CISEPA, pp. 123-155.

Vergara, A. (2001). "El Perú tras la elección imposible". Letras Libres, julio, pp. 68-70. 
